\title{
PILKADA SERENTAK TAHUN 2018: LEGACY SUMATERA UTARA
}

\author{
Oleh: \\ Shohibul Anshor Siregar \\ Dosen FISIP UMSU, Medan
}

Disampaikan pada:

Kuliah Umum pada FISIP UMTS

Padangsidimpuan, 24 Maret 2018 
I. PILKADA SERENTAK DAN KONDISI GEOGRAFIK SUMATERA UTARA 


\section{PEMILIHAN KEPALA DAERAH 2018 PEMILIHAN CUBERNUR}

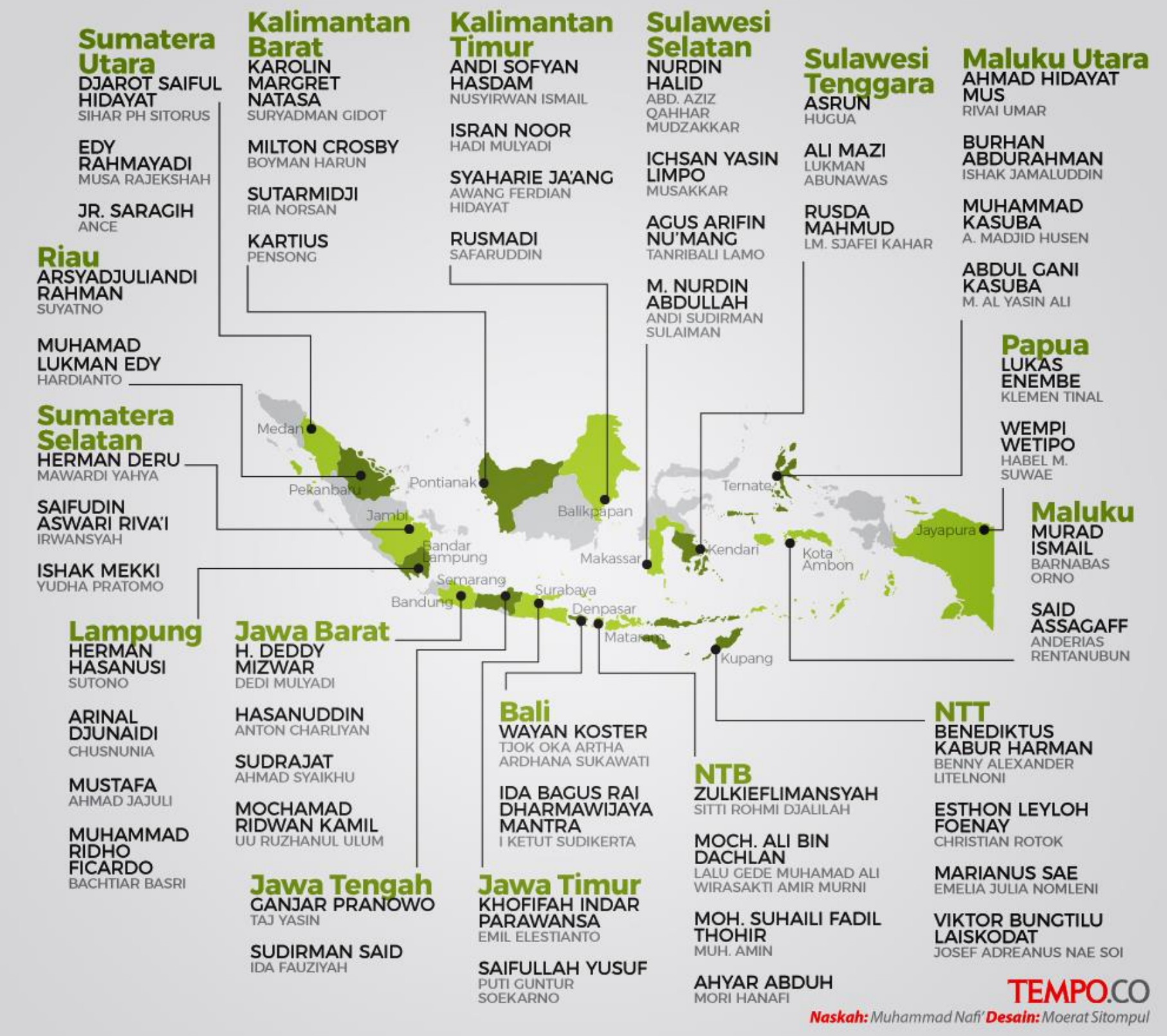


$-115.172 .000$

SIRAJUDIN PASKKALIS

$-94.180 .996$

TEMPOCO

PEMILIHAN

KEPALA DAERAH 2018

10 CALON

TERMISKIN

Salah satu syarat yang harus dipenuhi calon kepala daerah ketika mendaftar ke Komisi Pemilihan

Umum adalah berkas Laporan Harta Kekayaan

Penyelenggara Negara. Berkas itu dikumpulkan ke Komisi Pemberantasan Korupsi (KPK). Dari data KPK itu, ini 10 calon dengan harta paling sedikit.

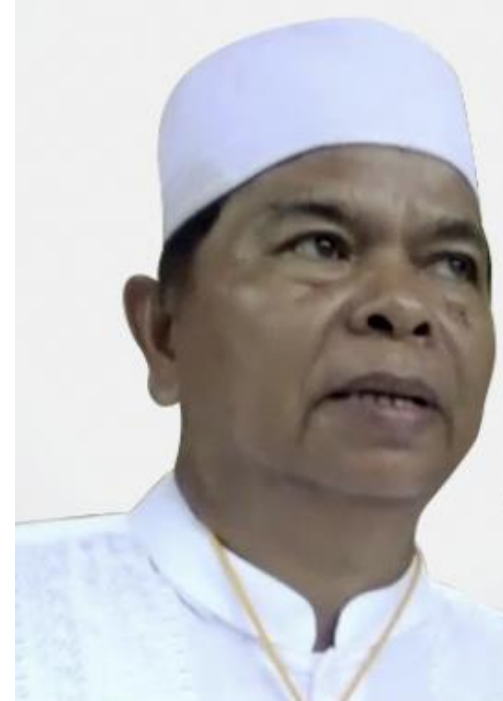

Syapuani, pensiunan

PNS, calon independen

di Murung Raya,

Kalimantan Tengah.
KOTABAUBAU

$\mathbf{5 . 0 0 0 . 0 0 0}$

MARKUS BOMIAI

10.832.415

BARITOTIMUR

14.820 .667

DENNY NURILHAMT

15.100 .000

KUBU RAYA

MARTINUSA.

22.000.000

KOTAPALANGKA RAYA

PATHUL MUNIR

23.184.900

JUSTINUS WIHELMUS NIAK NUMFOR

23.250.000

KENI IKOMOUU

26.250.000 
DATA PARTISIPASI PEMILIH 1999-2014

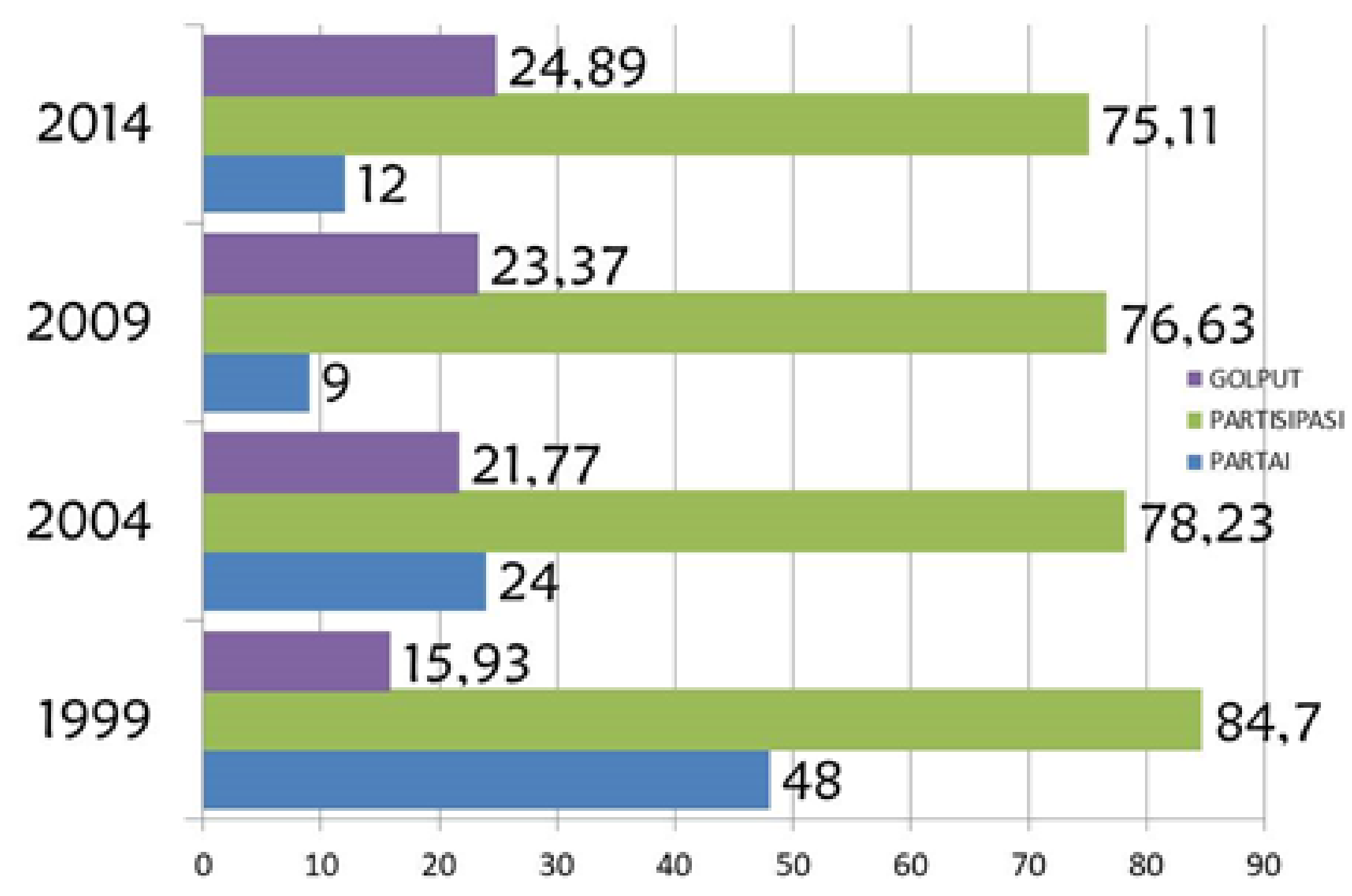




\section{KABUPATEN/KOTA, KONDISI DEMOGRASI DAN SOSIAL SUMATERA UTARA}

33 Kabupaten/Kota:

- 25 Kabupaten

- 8 Kota

\begin{tabular}{l} 
Pulau Nias \\
\hline 1 Nias \\
\hline 2Nias Utara \\
\hline 3Nias Barat \\
\hline 4 Nisel \\
\hline 5 Gusit \\
\hline
\end{tabular}

\begin{tabular}{l} 
Pantai Barat \\
\hline 1Madina \\
\hline 2 Tapsel \\
\hline 3 Palas \\
\hline 4 Paluta \\
\hline 5Tapteng \\
\hline 6 Sidimpuan \\
\hline 7Sibolga \\
\hline
\end{tabular}

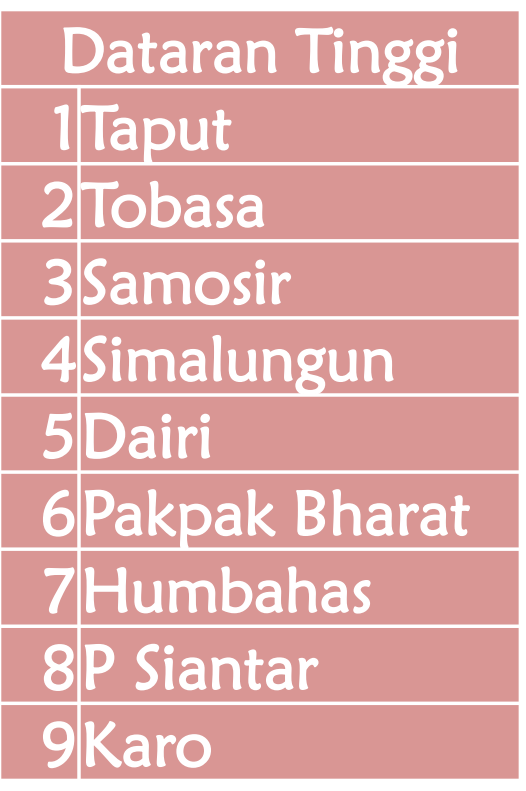

Pantai Timur

1 Labuhan Batu

2 Labuhan Batu Utara

3 Labuhan Batu Selatan

4Asahan

5Batubara

6Deli Serdang

7 Langkat

8Serdang Bedagai

9Tanjung Balai 10Tebing Tinggi

11 Medan

12 Binjai 


\section{PERSENTASE LUAS WILAYAH DAN PENDUDUK SUMATERA UTARA MENURUT KAWASAN (2016)}

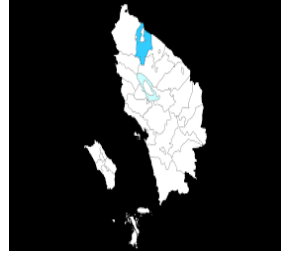

Luas Wilayah 72.981,23 Km2

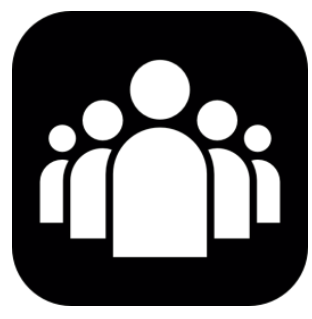

Jumlah Penduduk 14.102.911 Jiwa
Pulau
Nias
$7,71 \%$

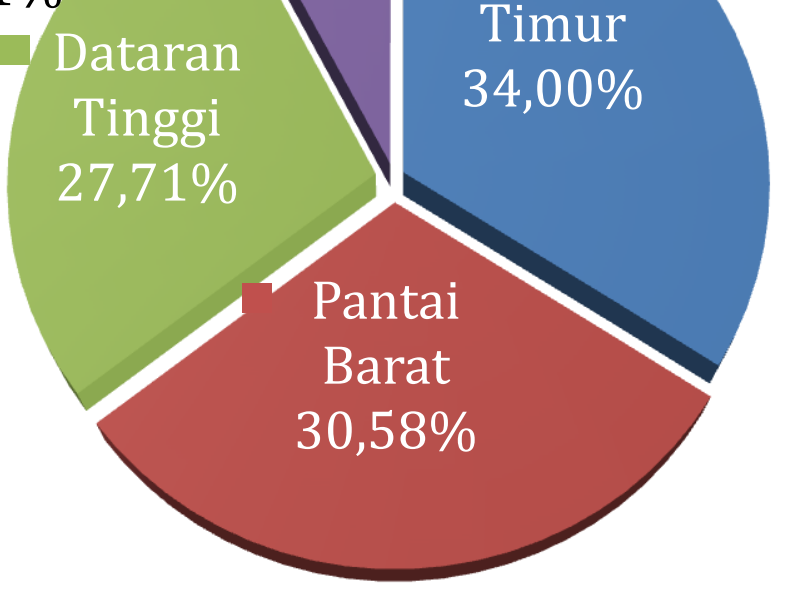

- Pulau Nias

$5,72 \%$

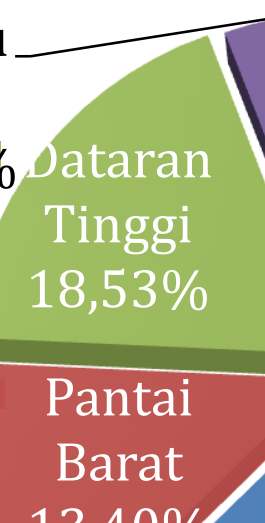

$13,40 \%$
- $\quad$ Penduduk

Kawasan

Pantai

Timur

62,35\%

dari total penduduk,

- Mereka mendiami 34,00\% dari total luas wilayah 


\section{AGAMA DAN SUKU BANGSA (SP 2010)}

\section{Agama}

\section{Suku Bangsa}
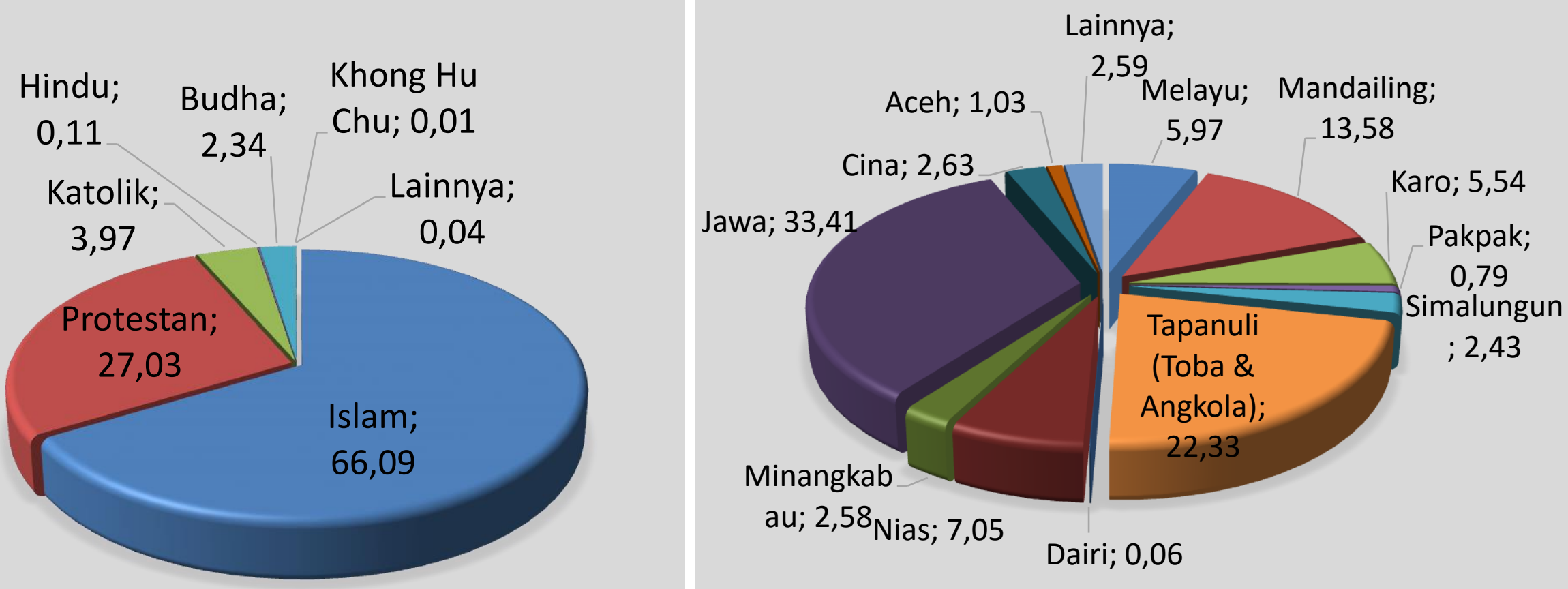


\section{IDI SUMUT (2016) 67,37. MENURUN 1.64 POIN DIBANDING TAHUN 2015. IDI SUMUT BERADA PADA URUTAN \\ TERENDAH KE DELAPAN SE-INDONESIA.}
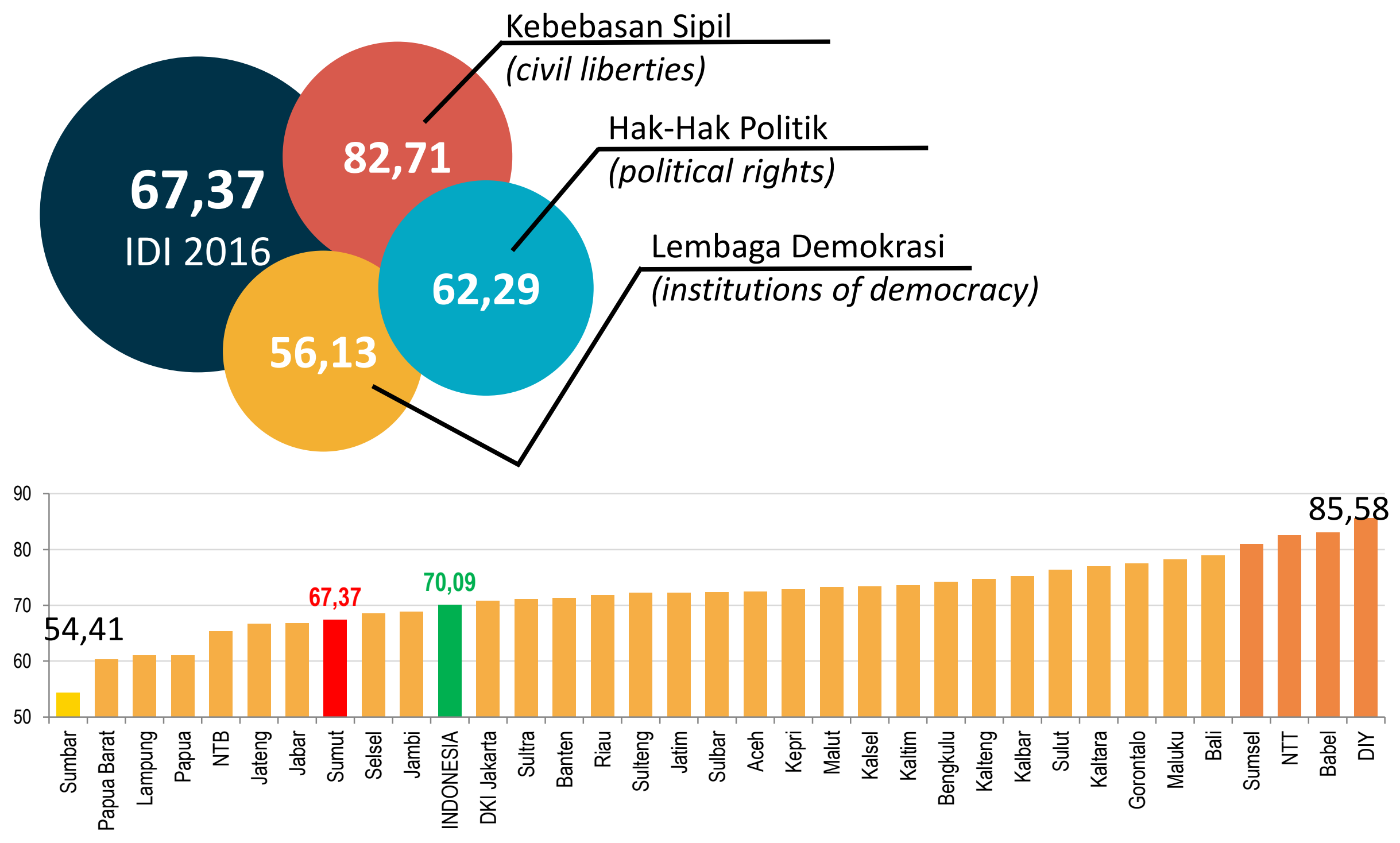


\section{II.PARA GUBERNUR DI PROVINSI SUMATERA UTARA}



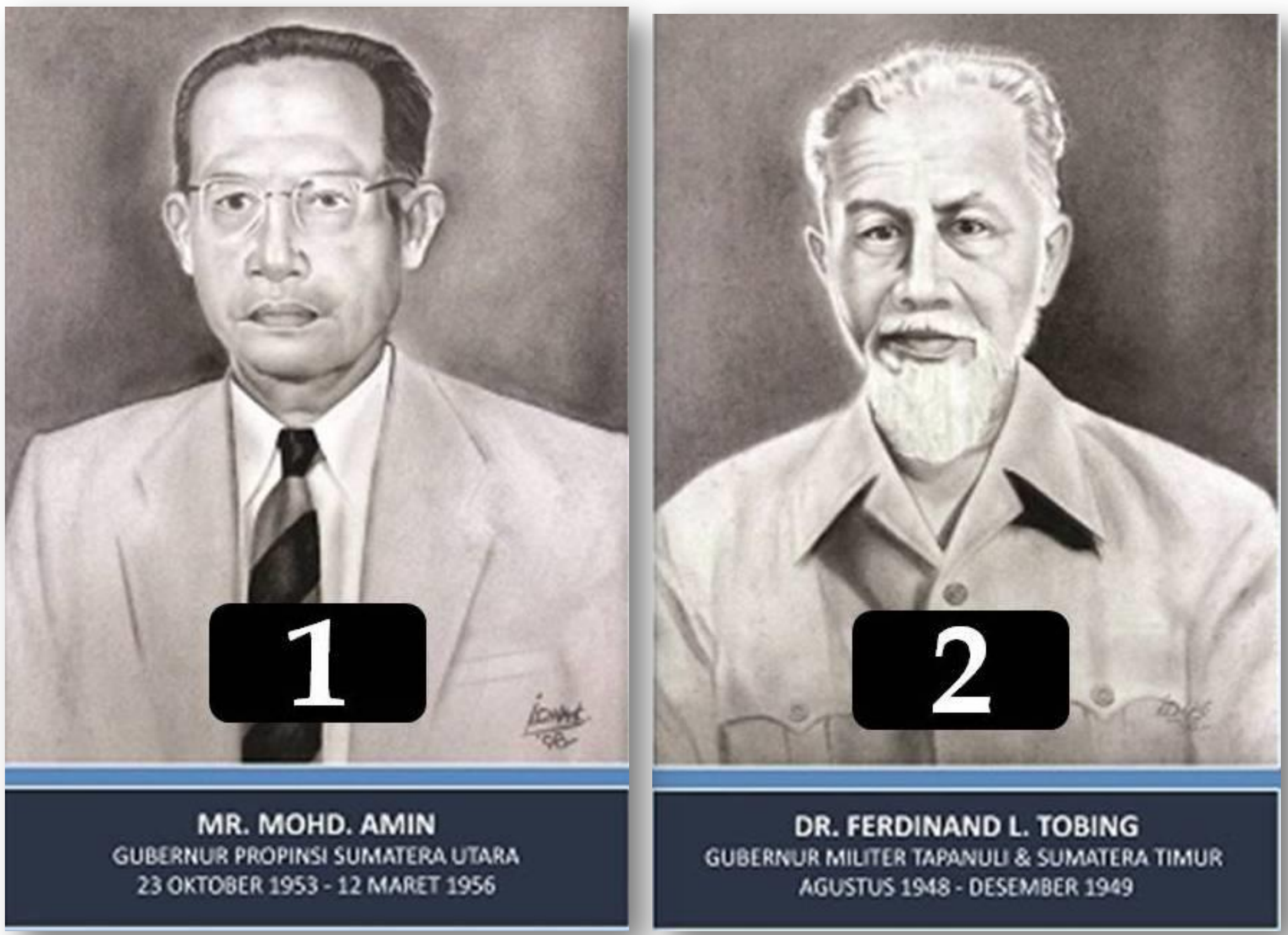

DR. FERDINAND L. TOBING

GUBERNUR MILTTER TAPANUU \& SUMATERA TIMUR AGUSTUS 1948 - DESEMBER 1969 

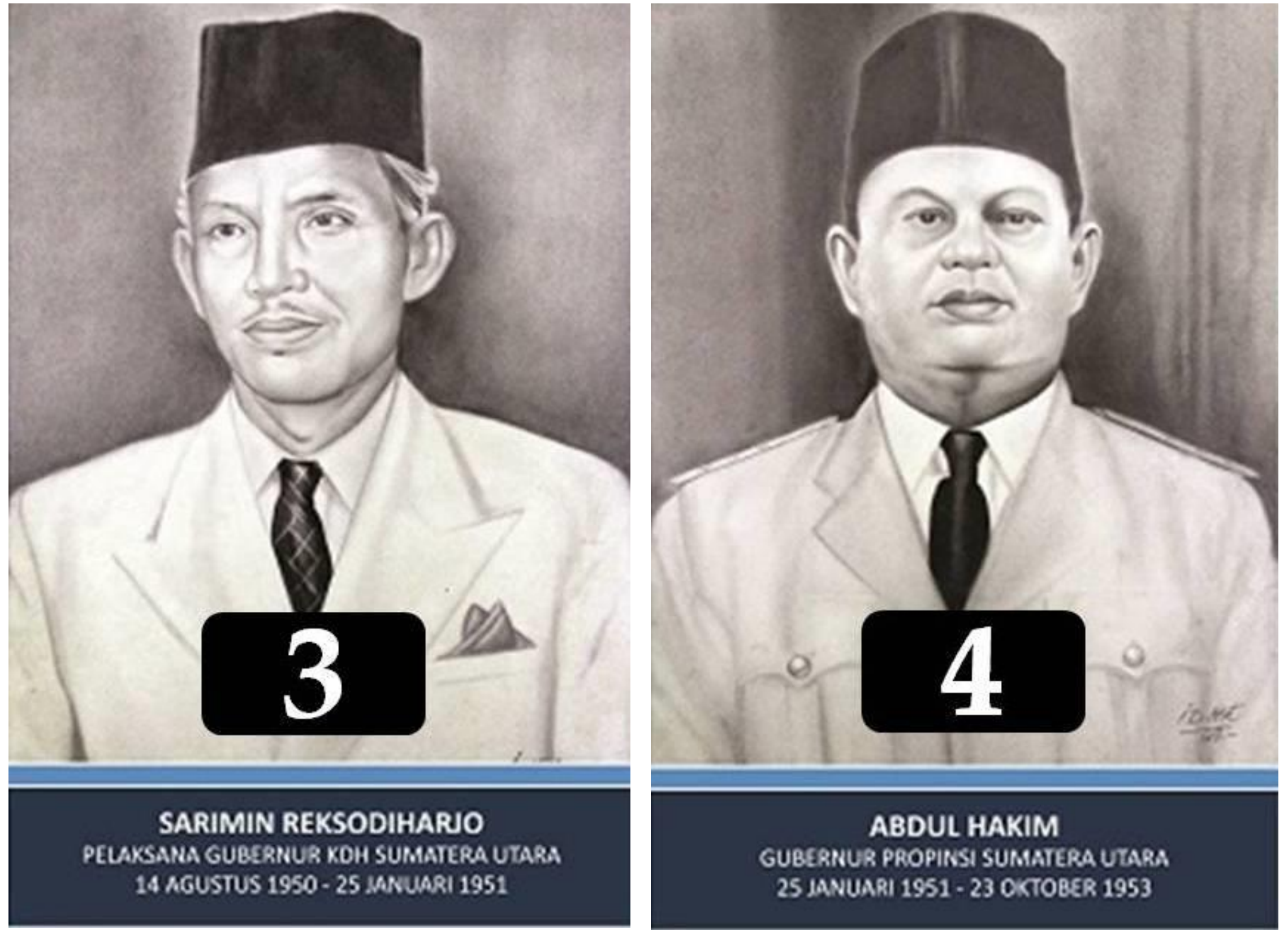


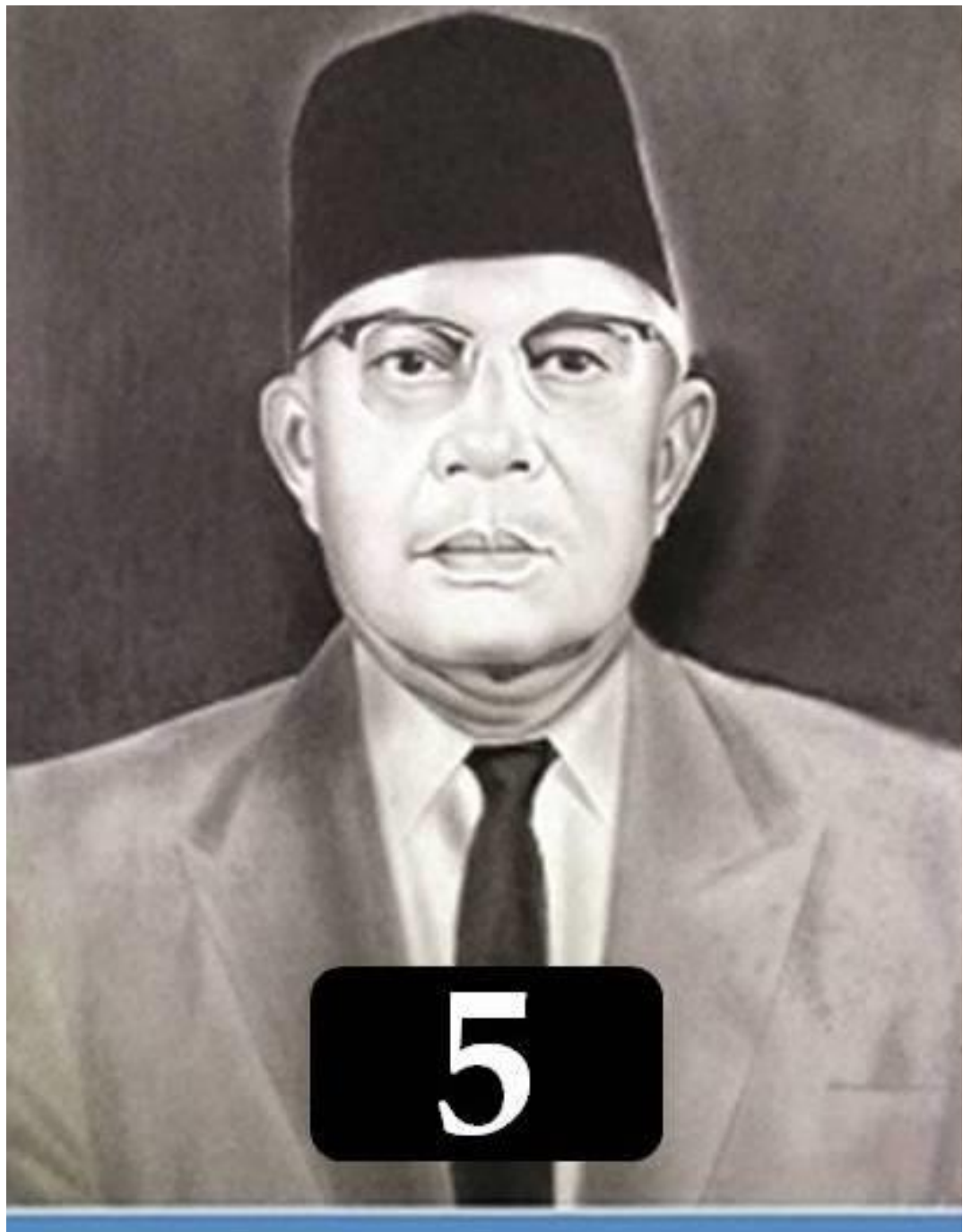

ST. KOMALA PONTAS

GUBSRRUR PROPINSI SUMATERA UTARA 18 MARET 1956 - 1 APRL 1960

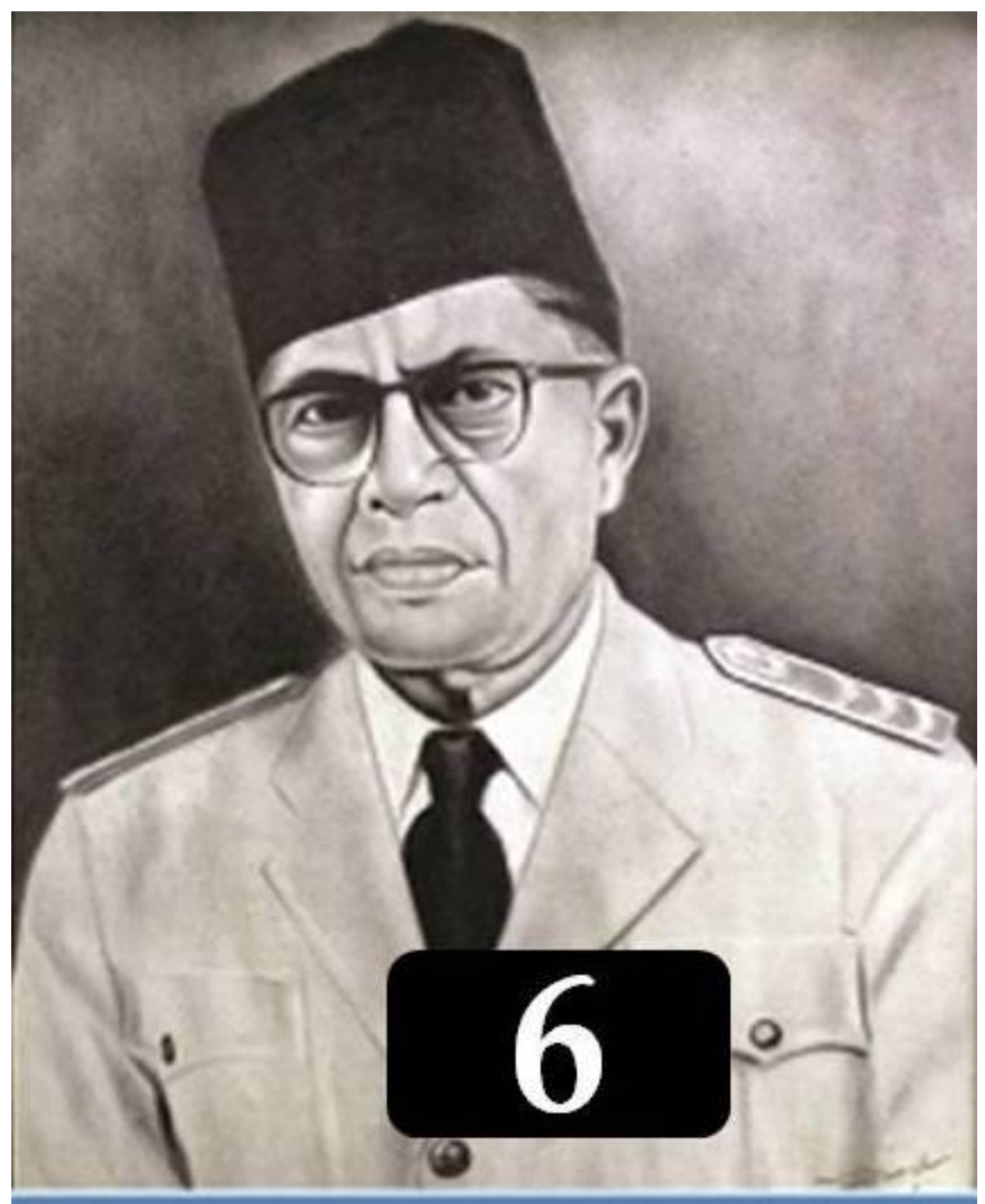

RANA JUNIUNGAN LUBIS GUBCRNUR PROPINSI SUMATERA UTARA 1 AORIL 1960 - 5 APRIL 1963 


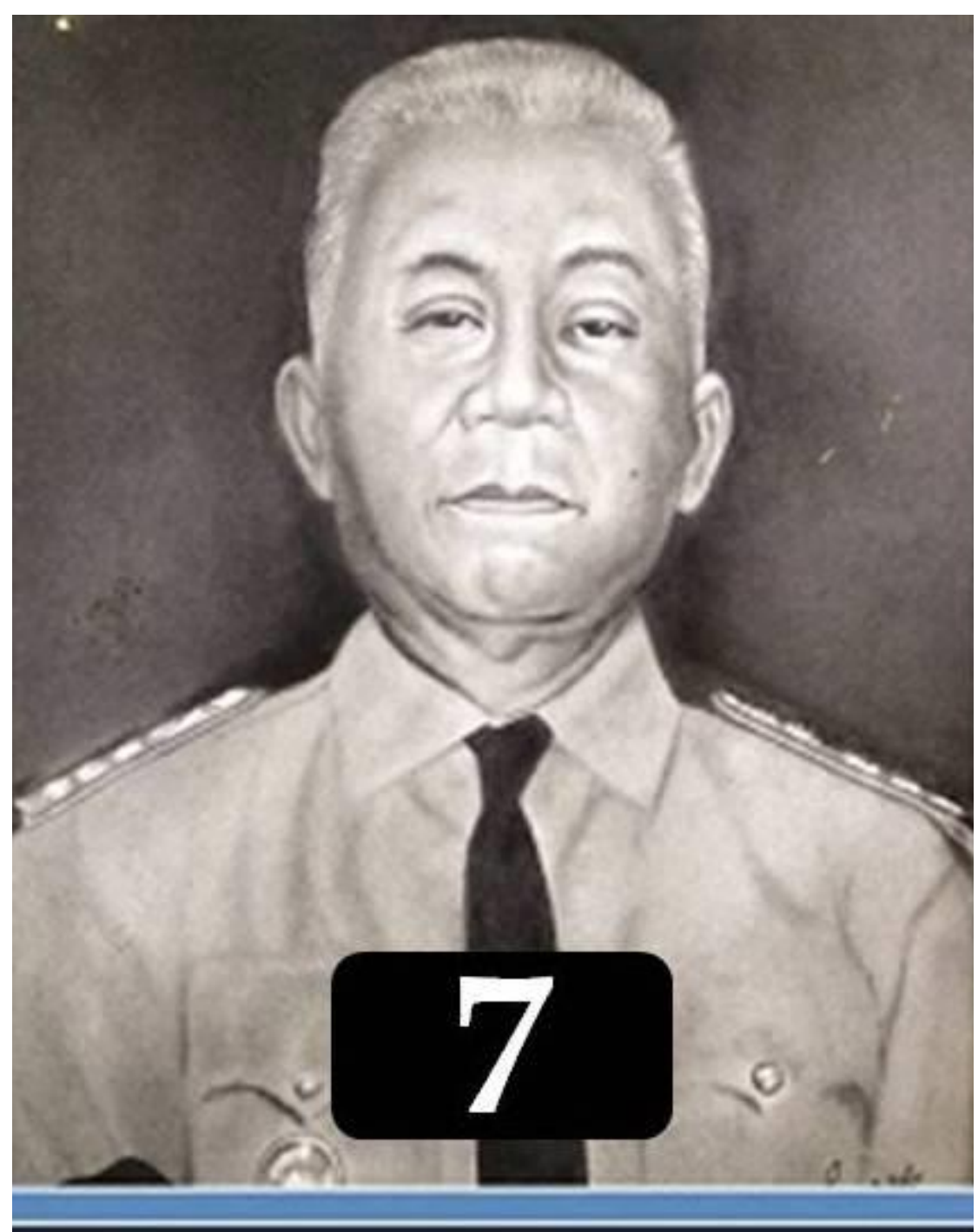

ENY KARIM

GUBERNUR KOH TINGKATI PROP SUMATERA UTARA SAPRIL 1963 - 15 JUU 1963

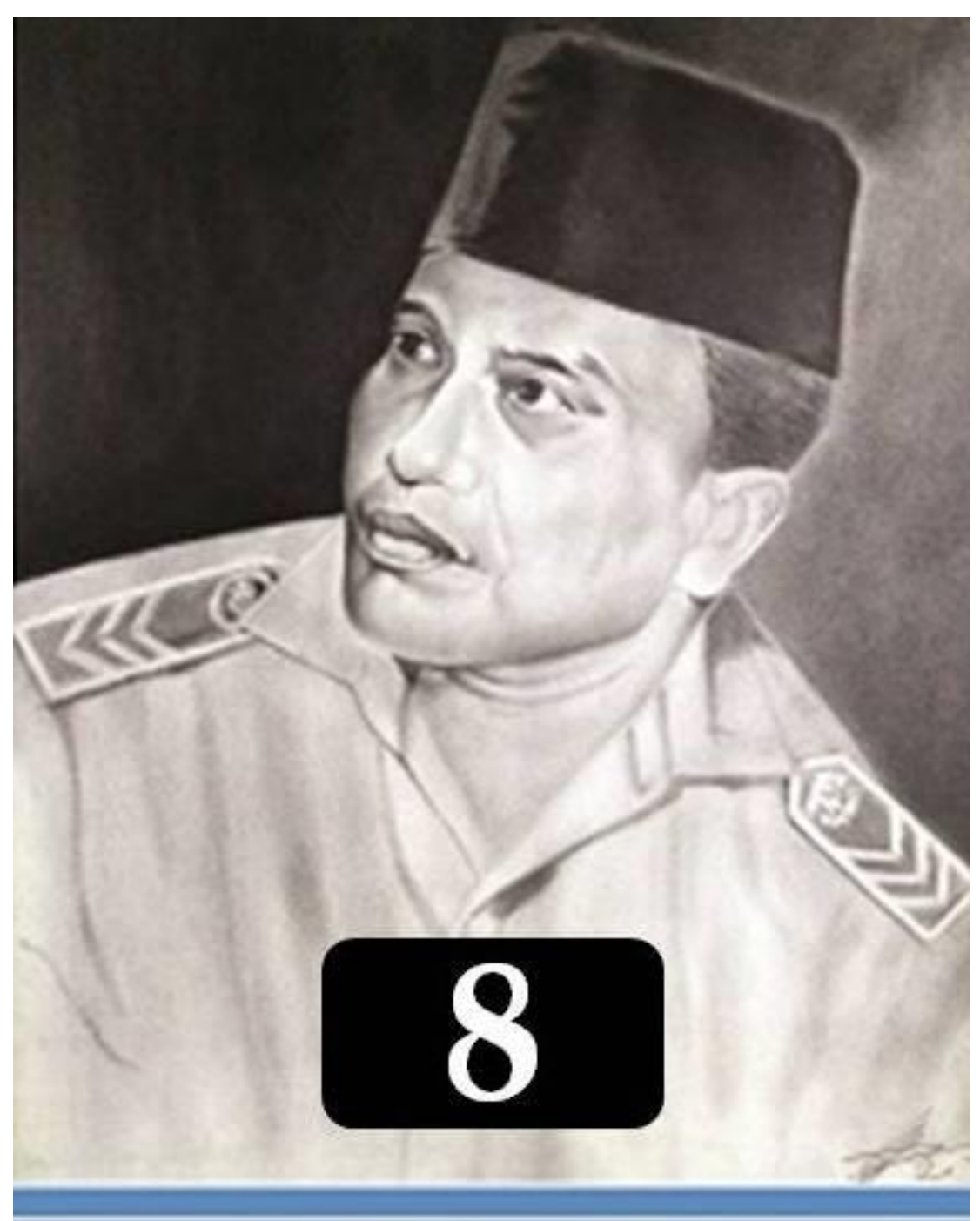

ULUNG SITEPU

GUBERNUR KOH TINGKAT I PROP SUMATERA UTARA 15 JULI 1963 - 16 NOPEMBER 1965 


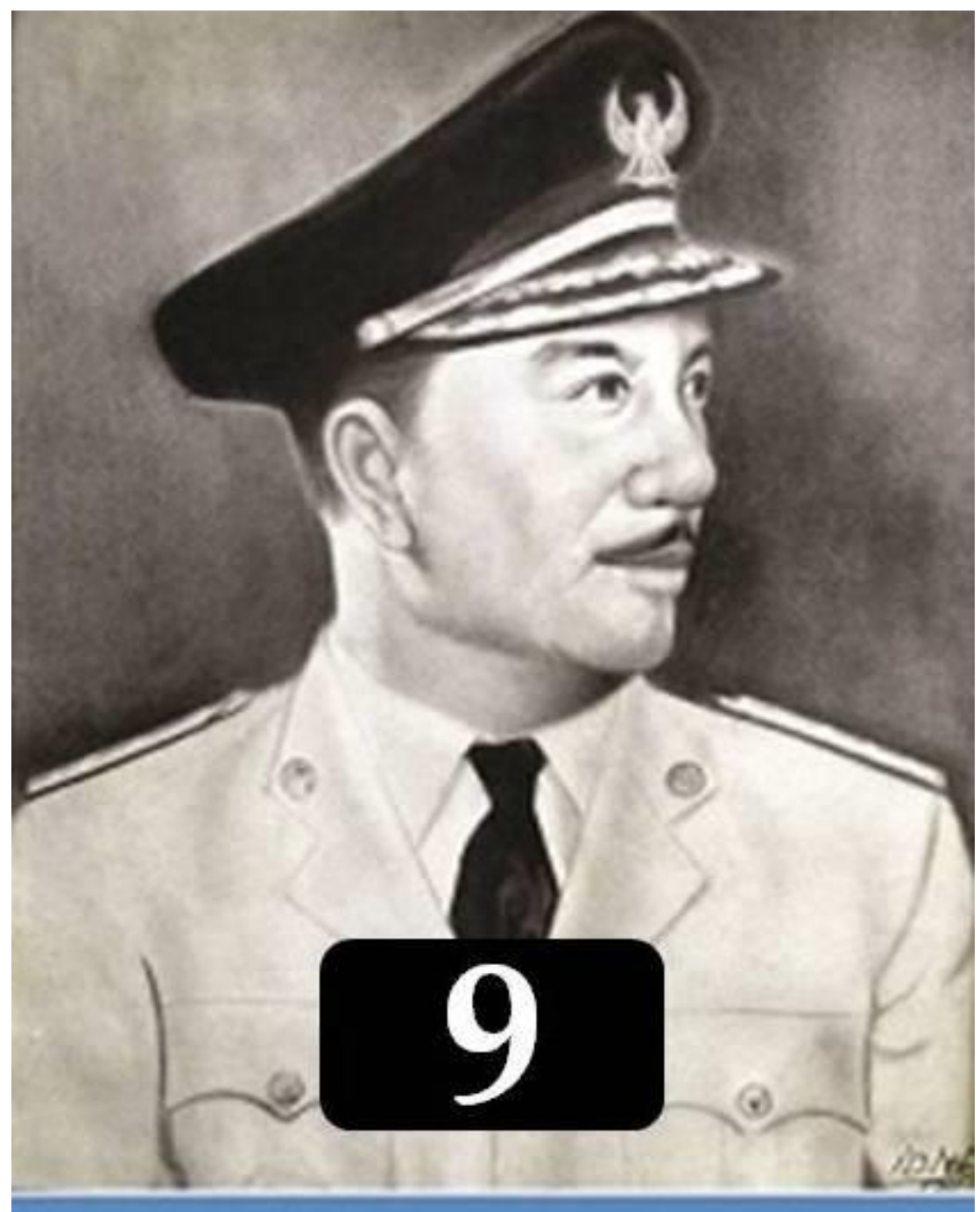

\section{P. R. TELAUMBANUA}

GUBERNUR KOH TINGKAT I PROP SUMATERA UTARA 16 NOPEMBER 1965 - 31 MARET 1967

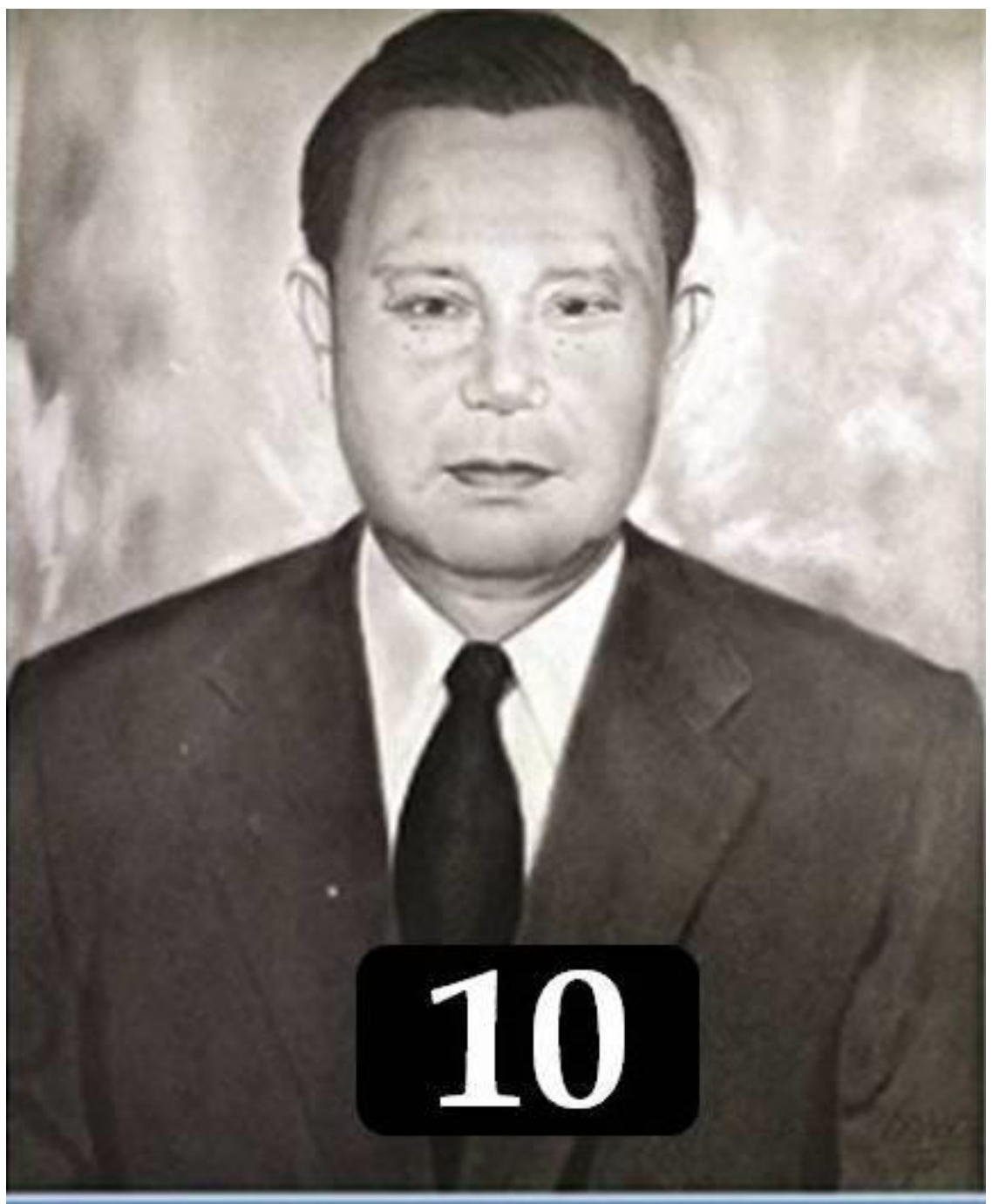

\section{MARAH HALIM}

GUBERNUR KOH TINGKAT I PRCP SUNATERA UTARA 31 MARET 1967 - 12 JURI 1978 


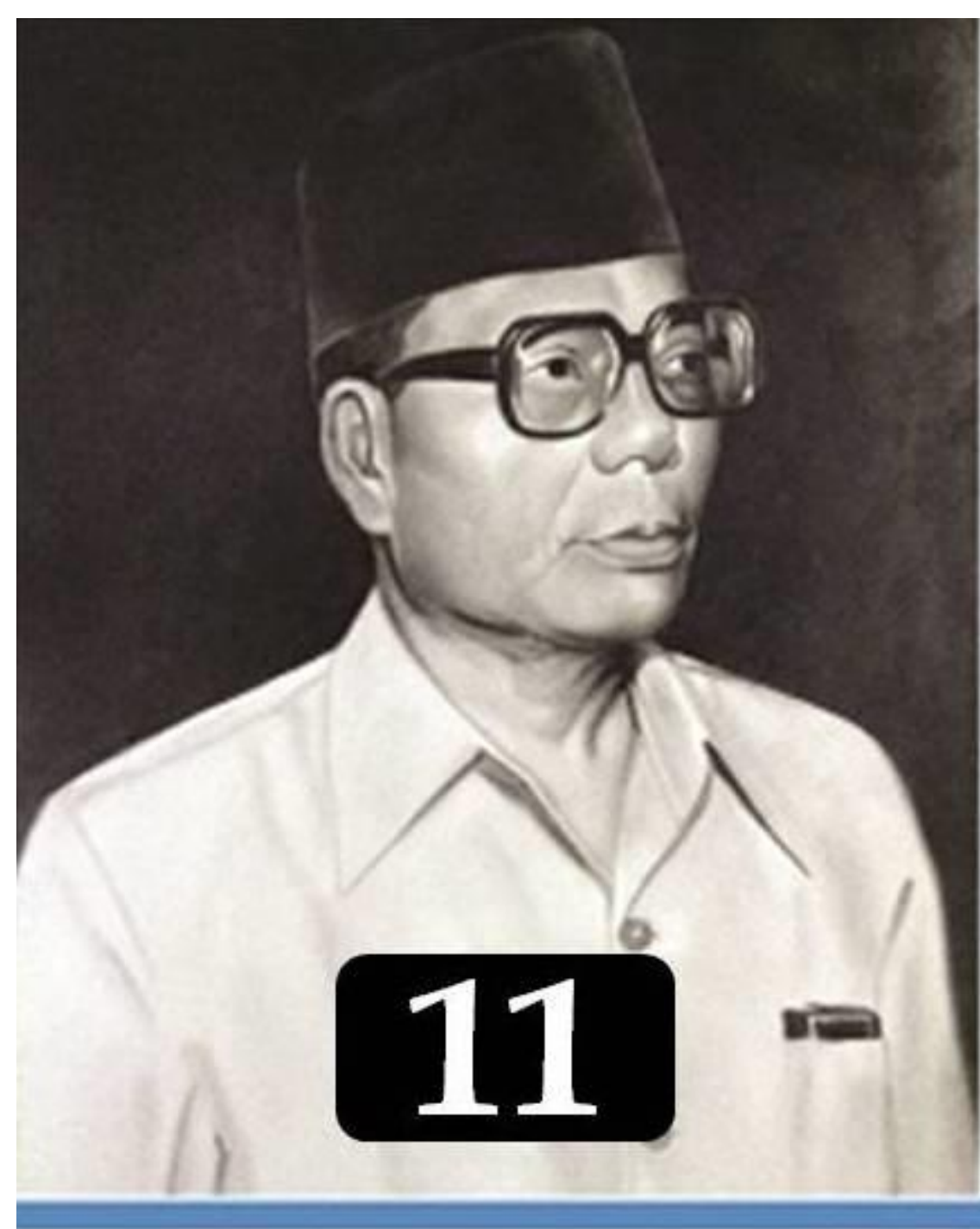

E. W. P. TAMBUNAN

GUBERNUR KOH TINGKAT I PROR SUMATERA UTARA 12 JUNI 1978 - 13 JUNI 1983

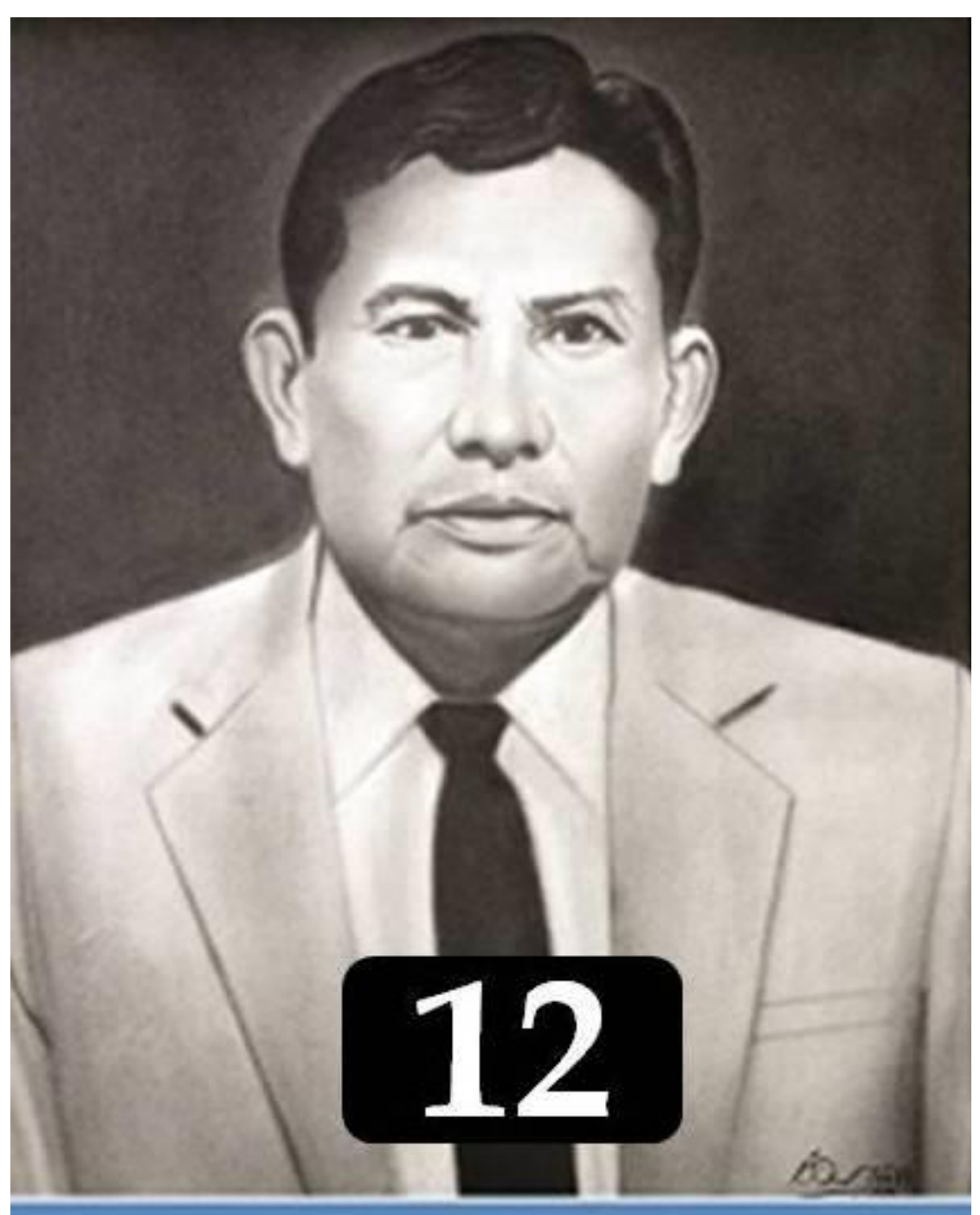

KAHARUDIN NASUTION

GUBERNUR KDH TINGKATI PROR SUMATERS UTARA 10 JUNI 1983 - 13 JUNI 1988 


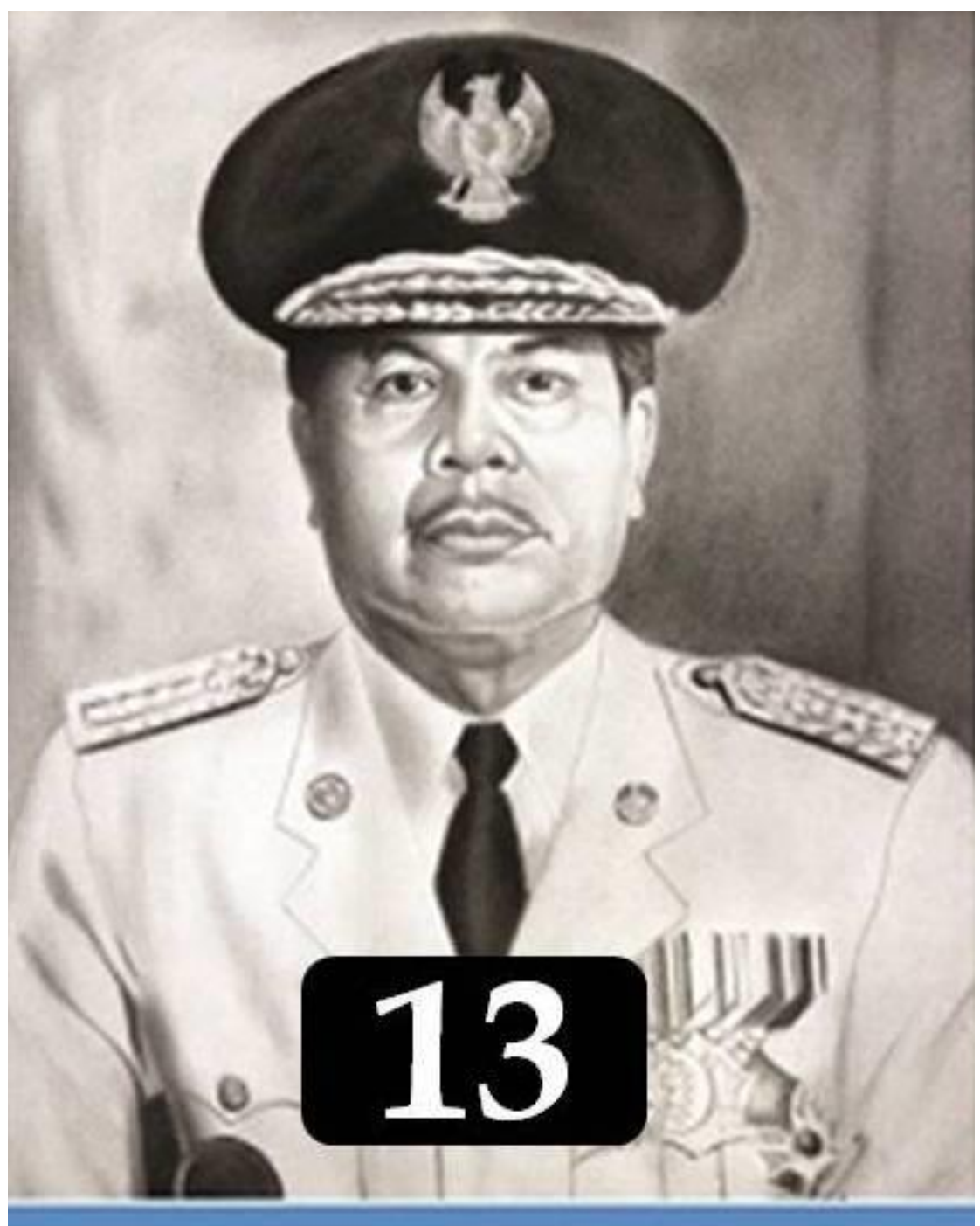

RAJA INAL SIREGAR

GUBERNUR KOH TINGKATI PROP SUNATERA UTARA 13 JUNI 1988 - 15 JUNI 1998

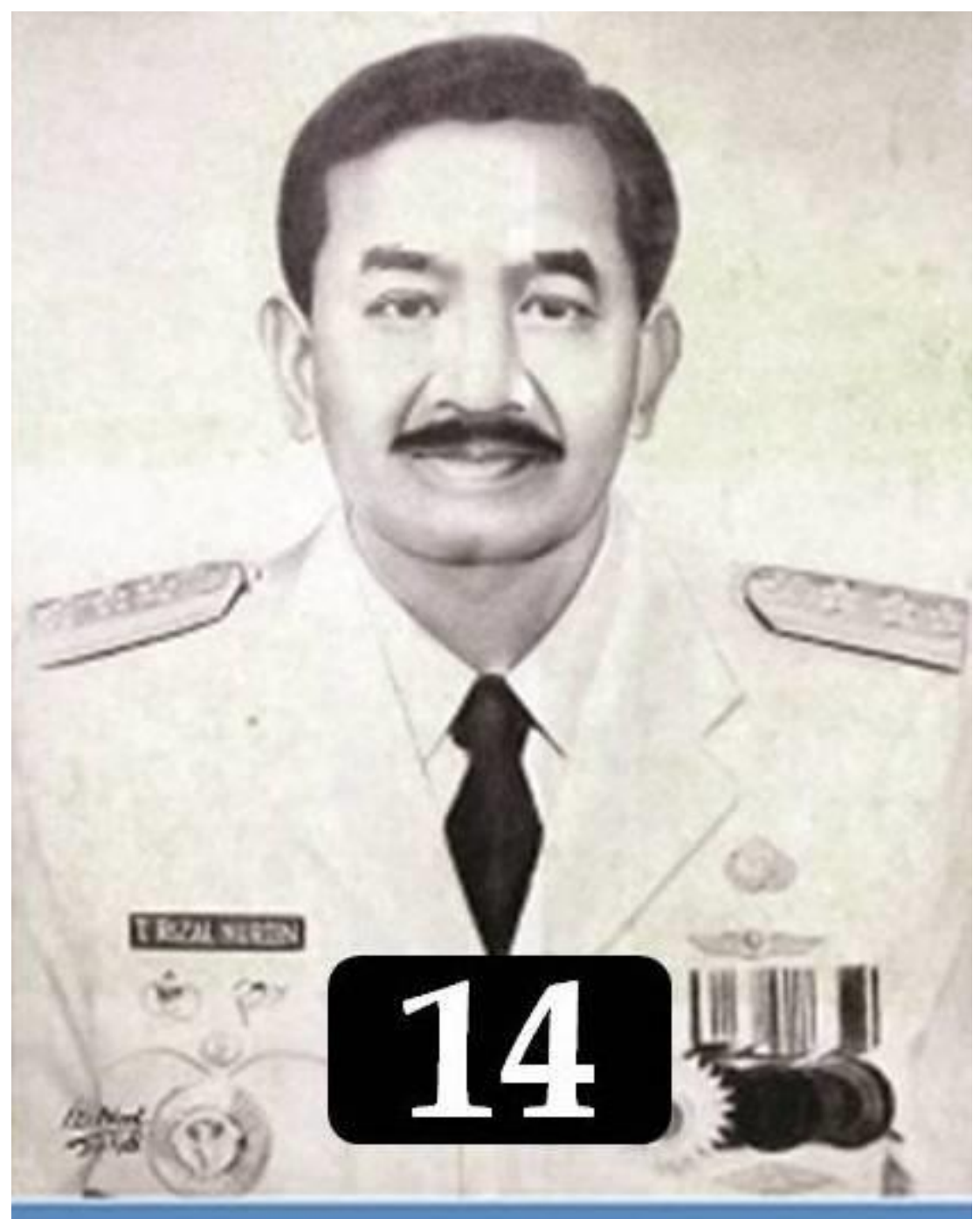

TENGKU RIZAL NURDIN

GUBERNUR KDH TINGKATI PROE SUMATERA UTARA 15 JUNI 1998 - 5 SEPTEMBBER 2005 

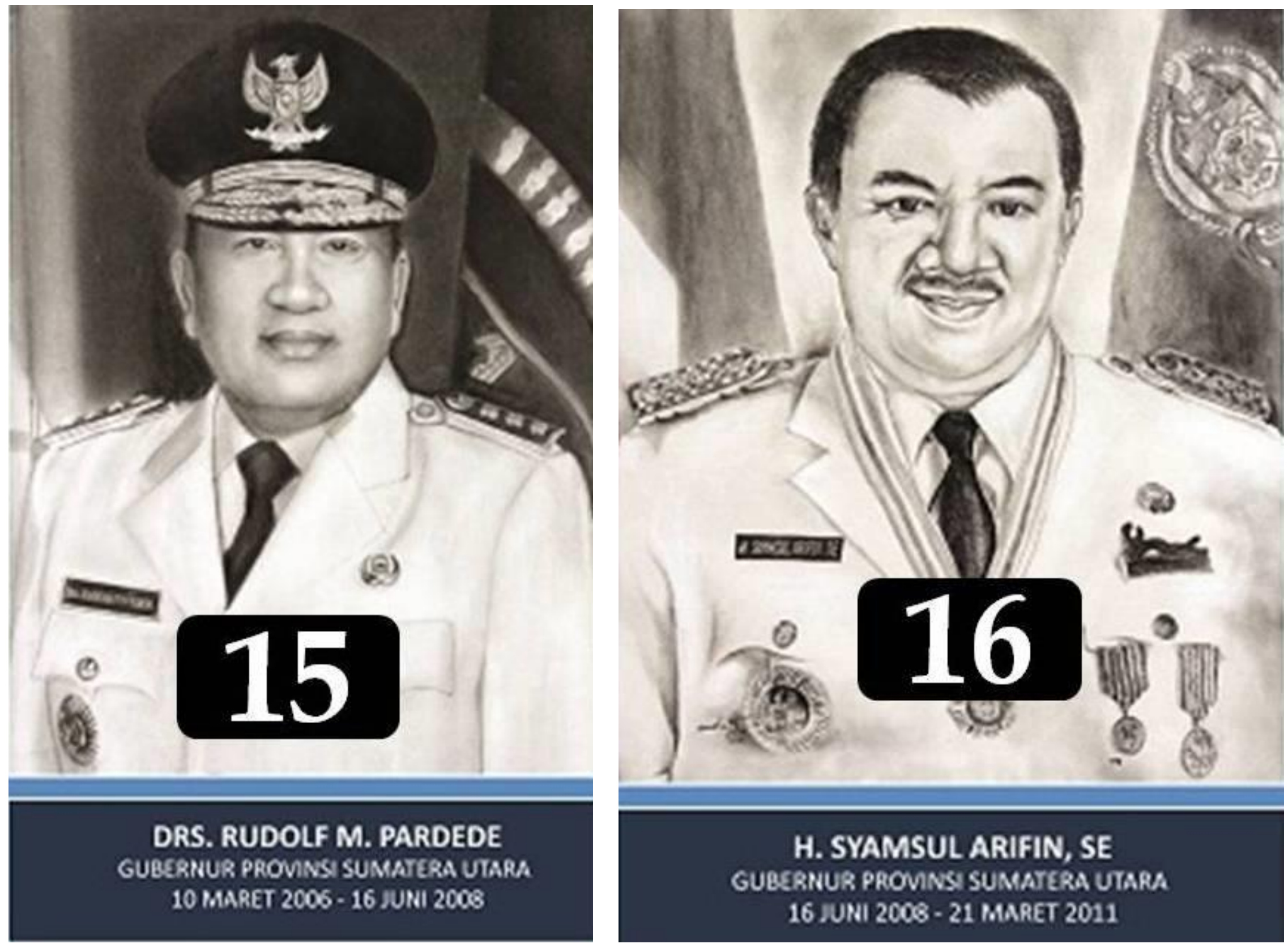

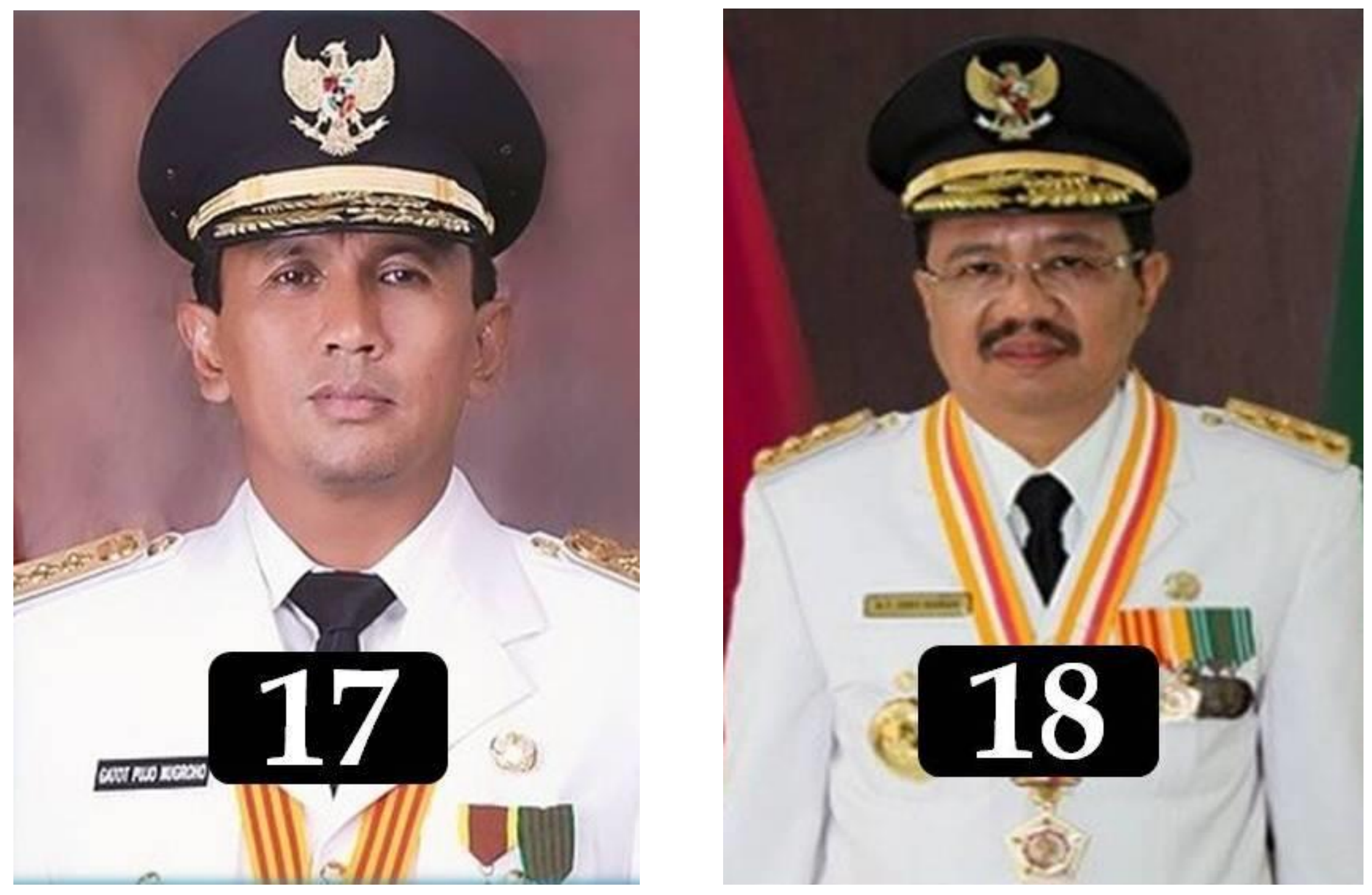


\section{III.TRANSISI DAN LEGACY}


TRANSISI POLITIK DAN PEMERINTAHAN

DI SUMATERA UTARA

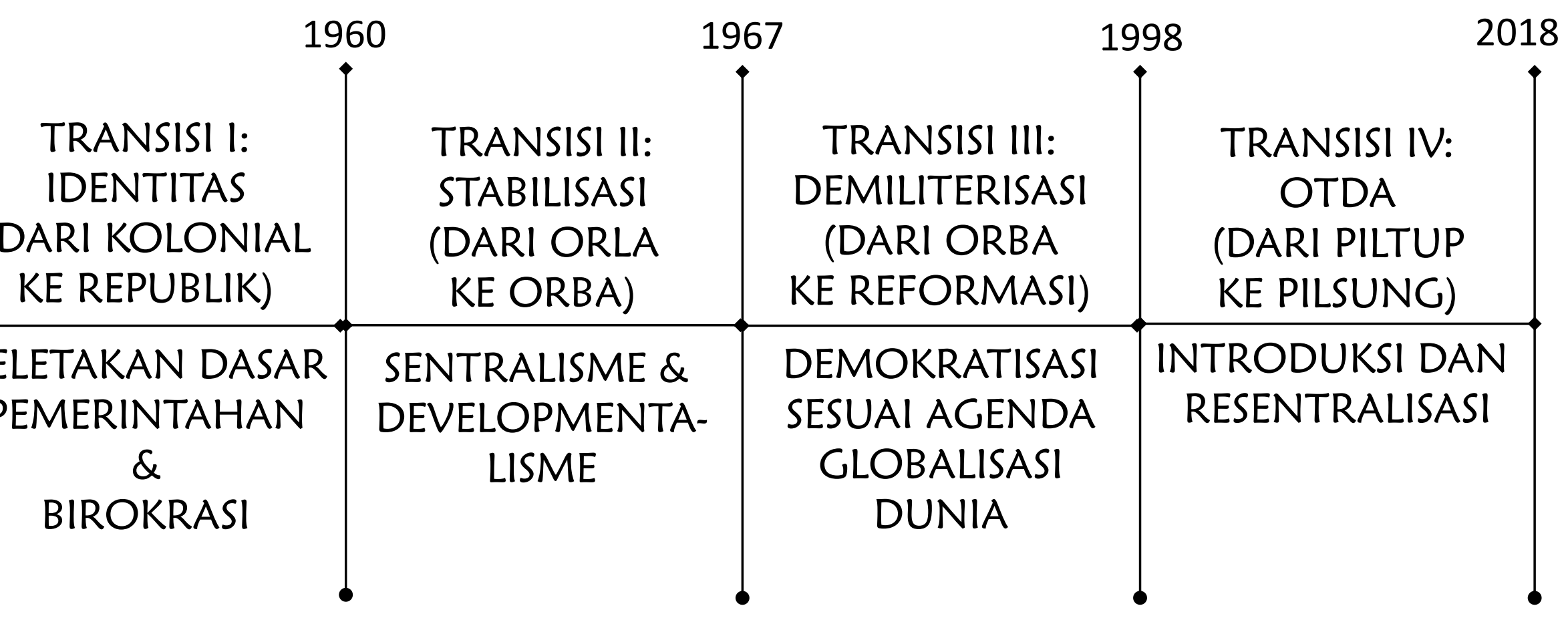

SM AMIN NASUTION,

FL TOBING,

SARIMIN REKSODIHARDJO, ABDUL HAKIM HARAHAP,

SM AMIN NASUTION,

RAJAMIN NASUTION

(SUTAN KUMALA PONTAS)
RADJA DJUNDJUNGAN LUBIS, MARAH HALIM HARAHAP, ENY KARIM, ULUNG SITEPU, PR TELAUMBANUA
EWP TAMBUNAN, KAHARUDDIN NASUTION, RAJA INAL SIREGAR
RIZAL NURDIN, RUDOLF PARDEDE, SYAMSUL ARIFIN GATOT PUJONUGROHO ERRY NURADI 
LAMA (BULAN) MEMERINTAH

GUBERNUR SUMATERA UTARA

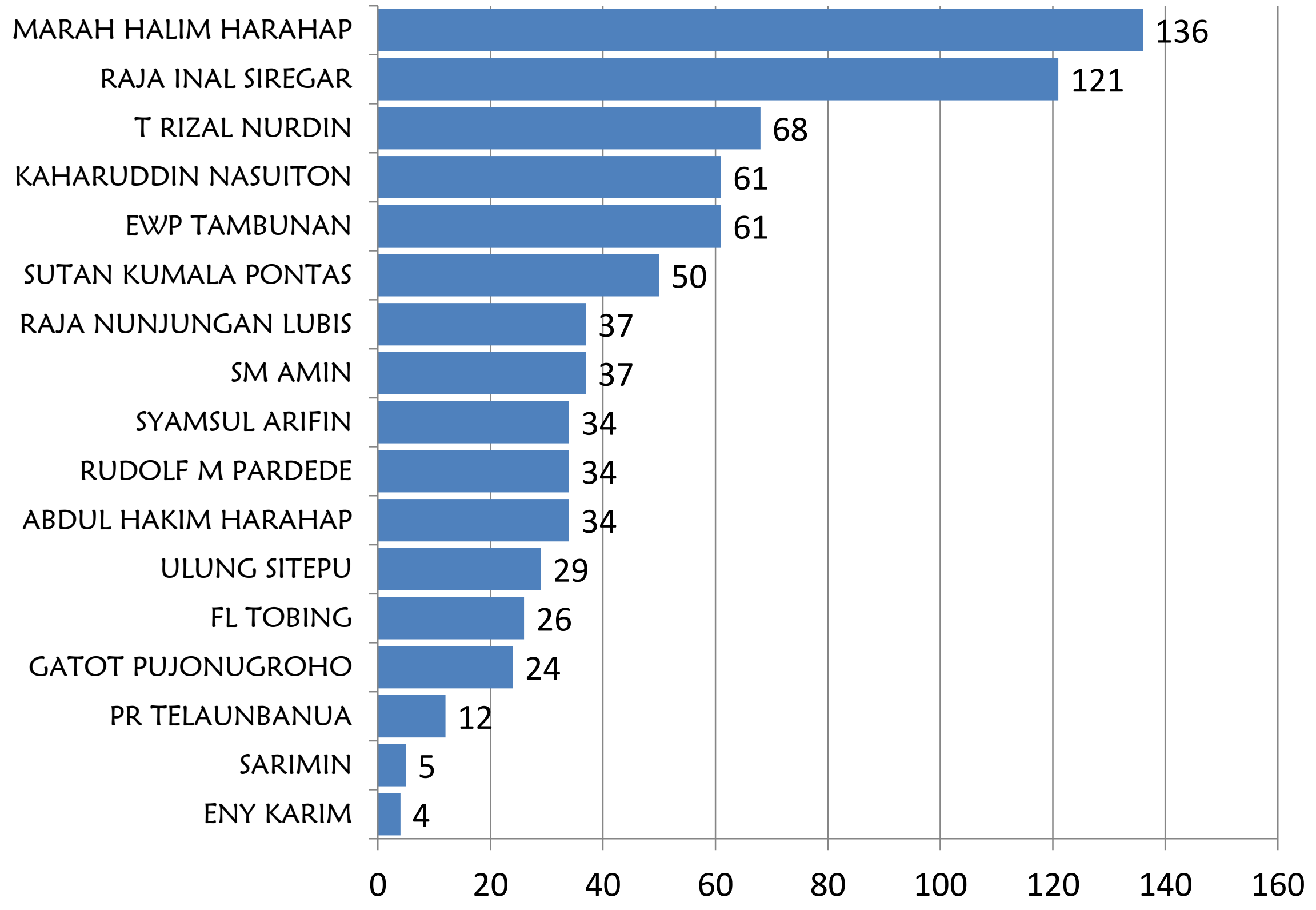




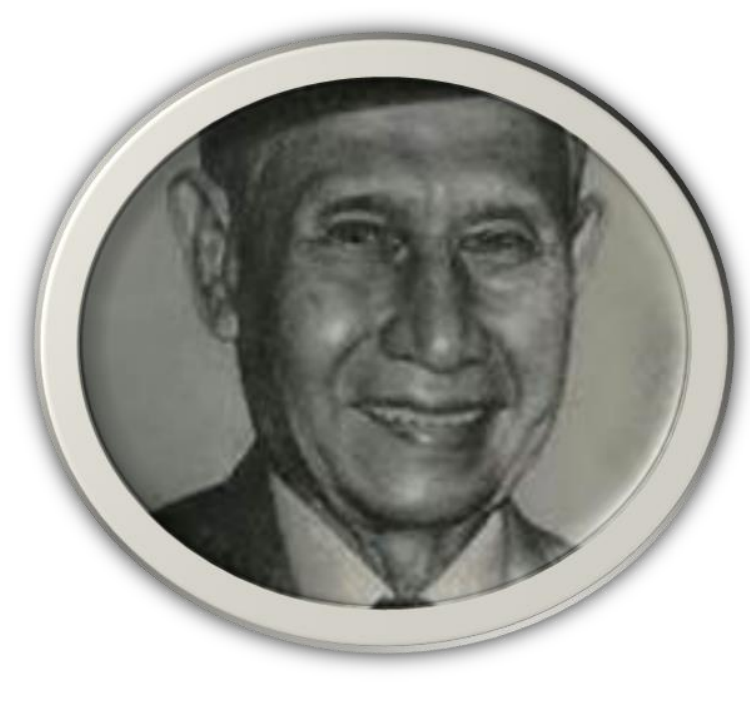

\section{GUBSU PERTAMA: SM AMIN}

SAAT PERANG KEMERDEKAAN BERKECAMUK, SM AMIN DIANGKAT SEBAGAI GUBERNUR MUDA SUMATERA UTARA PADA 14 APRIL 1947 OLEH GUBERNUR SUMATERA UTARA MR TEUKU MUHAMAD HASAN. INILAH YANC KELAK DIPANDANG SEBAGAI CIKAL-BAKAL PEMERINTAHAN PROVINSI SUMATERA UTARA.

BERHASIL MEMERTAHANKAN KEMERDEKAAN INDONESIA (MENGUNGSI) KE PEMATANGSIANTAR, PRESIDEN RI IR SOEKARNO LALU MENGANGKAT SM AMIN MENJADI GUBERNUR SUMATERA UTARA SECARA PENUH PADA 19 JUNI 1948.

SM AMIN NASUTION MEMIMPIN SUMUT 3 KALI DALAM PERTIKAIAN POLITIK DAN BERSENJATA YANG LUAR BIASA SULITNYA PADA AWAL REPUBLIK DIKONSTRUKSIKAN. BERBEDA DENGAN TOKOH PEMERINTAHAN MASA KINI,

SM AMIN ADALAH SEORANG YANG MENILAI PENTING MENEBAR PEMIKIRAN KEPADA MASYARAKAT LUAS MELALUI SEJUMLAH BUKUNYA. 


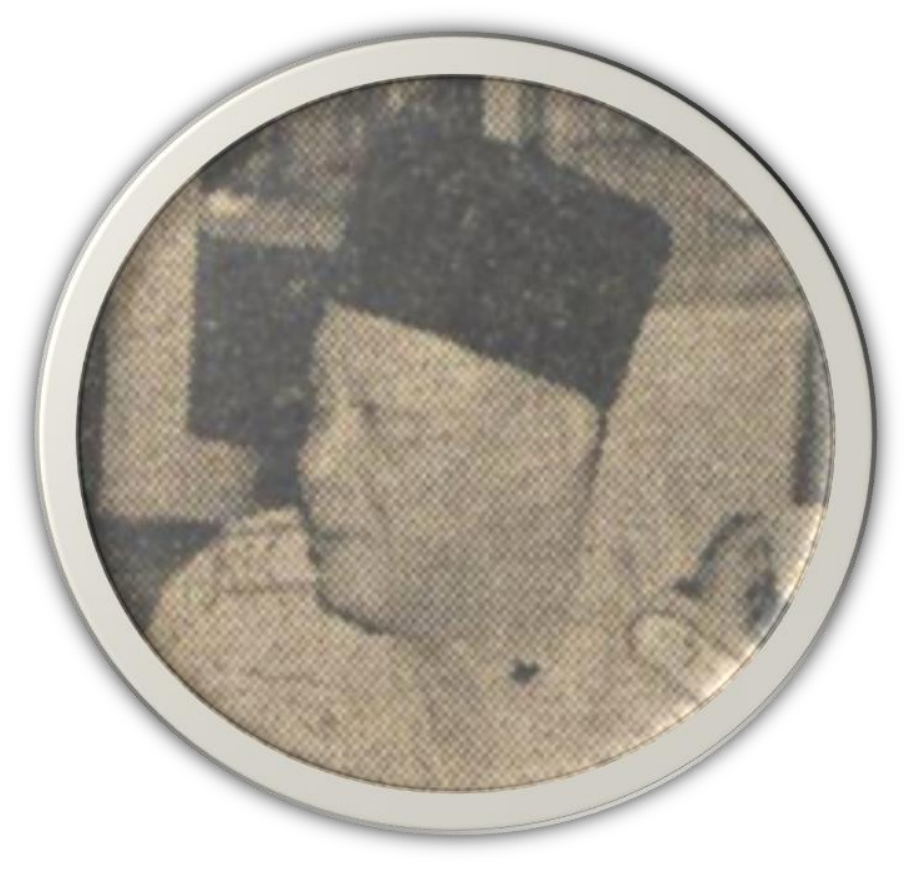

ABDUL HAKIM HARAHAP (GUBSU 1951-1953)

MEMBAWA PON III UNTUK DISELENGGARAKAN DI MEDAN. UNTUK ITU DIBANGUNLAH STADION TELADAN. SIAPA DI ANTARA KITA KINI YANG MAMPU MERAWAT, MISALNYA MENGECET DENGAN BAGUS DAN MENATANYA SELAYAK MUNGKIN?

ABDUL HAKIM MEMBANGUN WILAYAH EKSKLUSIF MEDAN BARU SEBELUM KITA KENAL PEMUKIMAN-PEMUKIMAN ELIT YANG SEKARANG.

ABDUL HAKIM MENSPONSORI PENDIRIAN USU YANG MENJADI KEBANGGAAN DAN SEKALIGUS PENGGEMBLENGAN KADER-KADER BANGSA.

ABDUL HAKIM JUGA PERNAH MENJABAT WAKIL PERDANA MENTERI (1950) 


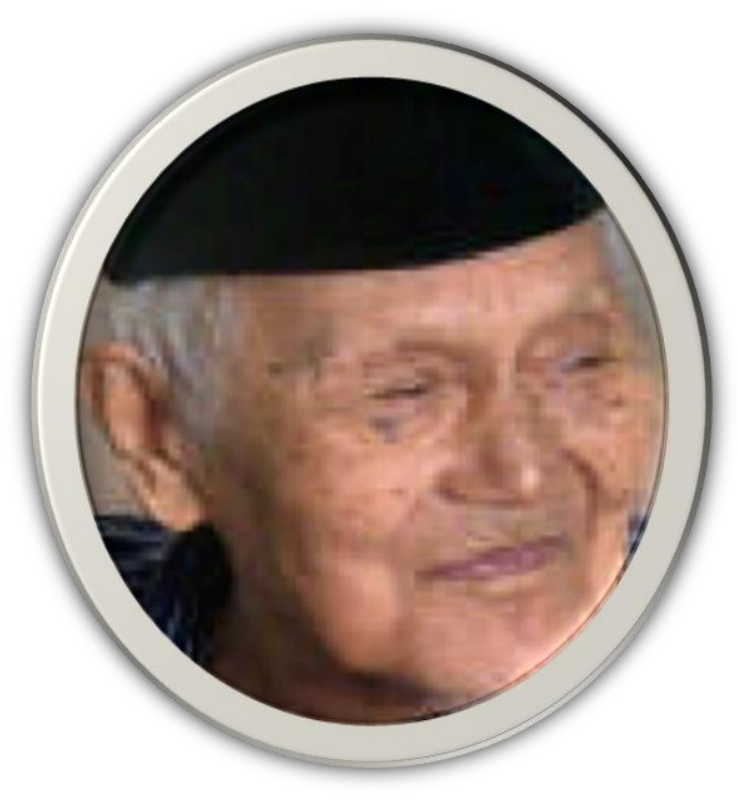

\section{MARAH HALIM HARAHAP \\ (1967-1978)}

ADALAH GUBSU TERLAMA (11 TAHUN). IA JUGA MENJADI MANTAN GUBSU BERUSIA TERPANJANG (MENINGGAL DUNIA SAAT USIA 94 TAHUN). SELAIN ITU MARAH HALIM HARAHAP ADALAH PEJABAT PERTAMA DI ANTARA 3 GUBSU YANG BERTUGAS SELAMA KEKUASAAN POLITIK ORDE BARU DI BAWAH KEPEMIMPINAN JENDERAL SOEHARTO.

MARAH HALIM HARAHAP MERINTIS UPAYA PEMBANGUNAN OLAHRAGA SEPAKBOLA MODERN INDONESIA DAN MEMASARKANNYA MELALUI MARAH HALIM CUP. EVENT ITU SELALU DIHADIRI OLEH KESEBELASAN-KESEBELASAN LUAR NEGERI. EVENT ITU TERDAFTAR SEBAGAI AGENDA FIFA.

IA MEMBANGUN JALAN DENGAN JAMINAN HANYA BOLEH MULAI RUSAK SETELAH 25 TAHUN. 


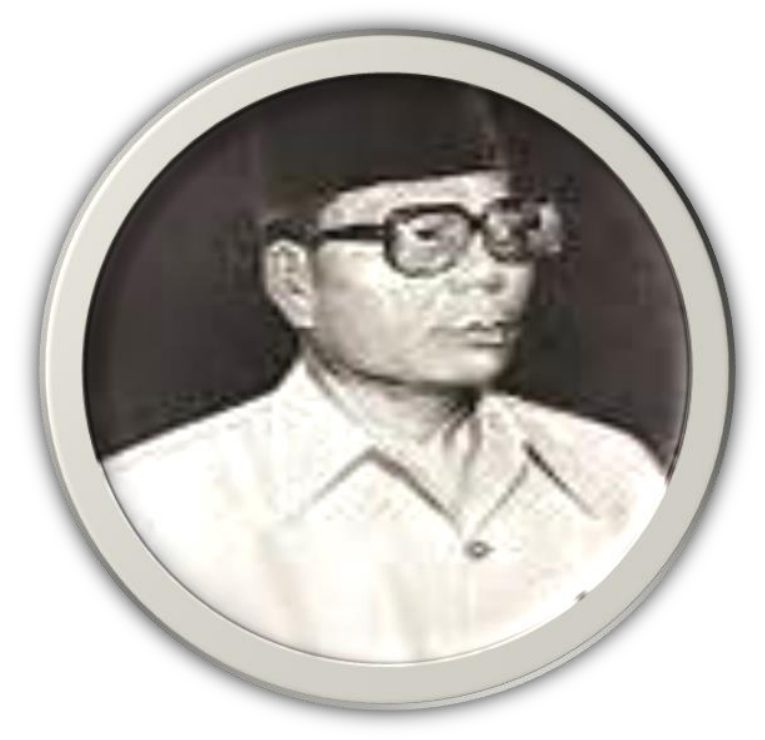

\section{EWP TAMBUNAN \\ (1978-1983)}

EWP TAMBUNAN ITU ORANG YANG BEROLEH EJEKAN SINIS DARI BANYAK ORANG PADA ZAMANNYA KARENA SELALU DENGAN TEGAS MENGEMBALIKAN (KE PUSAT) SETIAP ANGGARAN YANG TIDAK HABIS DIGUNAKAN.

SEKARANG POLA PENGHABISAN ANGGARAN DENGAN BERBAGAI KEGIATAN YANG TAK MASUK AKAL DIRESTUI BERSAMA OLEH EKSEKUTIF, LEGISLATIF DAN JUDIKATIF BERIKUT SELURUH KOMPONEN PENEGAKAN HUKUM YANG ADA, BAIK DI PUSAT MAUPUN DI DAERAH.

SEMUA TAHU KEGIATAN MENGHABISKAN ANGGARAN MENJELANG AKHIR TAHUN ANGGARAN ITU HANYA MODUS PENCURIAN UANG NEGARA, TETAPI MALAH TETAP SAJA DISENANGI. 


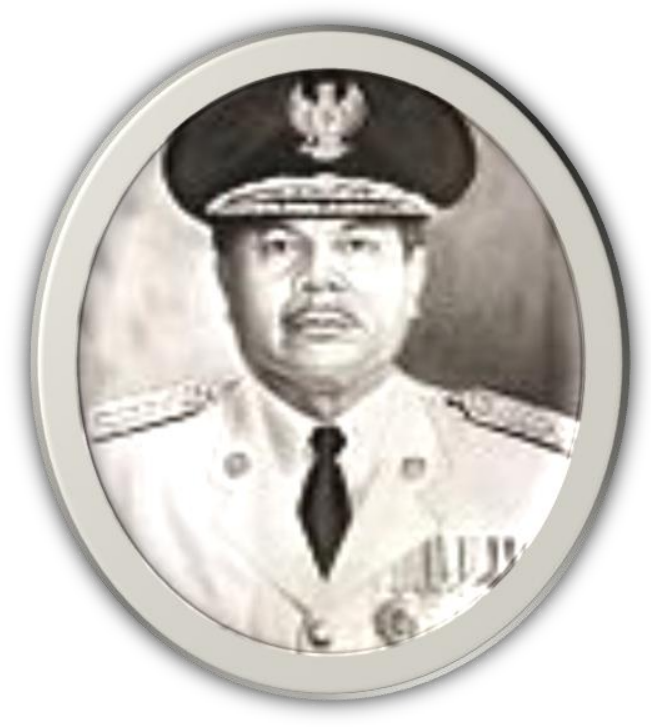

\section{RAJA INAL SIREGAR (1988-1998)}

SEORANG PENGGEBRAK. DULU ADA PEJABAT YANG MENYEBUT BAHWA MARTABE ITU ADALAH AKRONIM (SINGKATAN) DARI MARKISA TERONG BELANDA. BAGINYA MARTABE ADALAH SEJENIS MINUMAN KHAS BERUPA JUICE.

MARTABE (MARSIPATURE HUTA NA BE) YANG JUGA DIADOPSI DALAM BERBACAI BAHASA LOKAL DI SUMUT (KARO MISALNYA MENYEBUT PESIKAP KUTA KEMULIHENTA) DICANANGKAN DI TANJUNG IBUS (LANGKAT, 1981) KURANG LEBIH SEBACAI SEBUAH REAKSI POLITIK DAN BENTUK KETAK-SABARAN ATAS PERGILIRAN JATAH BERDASARKAN SISTEM POLITIK PEMBANGUNAN NASIONAL YANG TAK ADIL.

MARTABE ADALAH SEBUAH PERLAWANAN UNTUK KEPENTINGAN PEMBANGUNAN EKONOMI KERAKYATAN, SEBAGAIMANA PADA WAKTU SEBELUMNYA ADA JUGA PROGRAM YANG MIRIP DIBERINAMA MADUMA (BHS TAPANULI, MAKIN SEJAHTERA). 


\section{IV.PENUTUP}

BANYAK ORANG HEBAT PERNAH MEMERINTAH DI SUMATERA UTARA DENGAN CATATAN LEGACY YANG LUAR BIASA.

SEMOGA SUMATERA UTARA SEMAKIN BAIK KE DEPAN 